\title{
Stress concentration around irregular holes using complex variable method
}

\author{
K R Y SIMHA and S S MOHAPATRA \\ Department of Mechanical Engineering, Indian Institute of Science, Bangalore \\ 560012 , India \\ e-mail: [simha,sekhar]@ mecheng.iisc.ernet.in
}

MS received 2 April 1998

\begin{abstract}
Engineering materials are vulnerable targets for damage by chemical agents. This results in various types of irregular cavities which may subsequently change their shape under the combined action of loads and chemical attack. Such shape evolutions are subject to certain constraints. This paper explores the evolution in stresses as a result of an evolution in the shape of an isolated irregular hole in an infinite elastic plate subjected to remote uniform stress. The constraint employed here is a fixed area for the irregular hole with variable perimeter as a result of the evolution. Increase in perimeter implies decrease in strain energy on account of increased surface energy. Such phenomena could also occur in polymeric sheets on account of viscoelasticity even in the absence of chemical agents. This paper presents the evolution in boundary stresses as the cavity evolves to take different shapes. Complex variable methods are developed to tackle three cases of remote loading: (a) hydrostatic tension, (b) uniaxial tension, and (c) pure shear state. Of the above three cases, the first case of hydrostatic loading leads to a remarkably simple result for the boundary stress as shown in this paper. The last case is obtained by superposing a uniaxial tension and uniaxial compression along orthogonal directions.
\end{abstract}

Keywords. Stress concentration; irregular holes; complex variables; conformal mapping.

\section{Introduction}

The complex variable approach gives elegant mathematical relations involving the unknown stresses and displacements. The three basic equations of plane elasticity in complex variables are given by the three Kolosov-Muskhellishvilli relations as follows

A list of symbols is given at the end of the paper 
(England 1971),

$$
\begin{aligned}
& \sigma_{x}+\sigma_{y}=4 \operatorname{Re} E^{\prime}, \\
& \sigma_{y}-\sigma_{x}+2 i \sigma_{x y}=2\left[\bar{z} E^{\prime \prime}+F^{\prime \prime}\right], \\
& 2 G(u+i v)=\kappa E-z \bar{E}^{\prime}-\bar{F}^{\prime},
\end{aligned}
$$

where $E, F=$ complex potentials of the complex variable $z=x+i y$, and $\kappa=(3-v) /$ $(1+v)$ for plane stress and $3-4 v$ for plane strain.

\section{Conformal mapping}

Conformal mapping is an operation in complex mathematics which maps a set of points in one coordinate system to a corresponding set in another, keeping the angle of intersection between two curves constant. This is widely used in solving elasticity problems.

\subsection{Introduction to conformal mapping}

Any mapping of a set of points $\zeta$ in the plane $\xi \eta$ to a set of points $z$ in the plane $x y$ is a correspondence that is defined for each point in the $\xi \eta$ plane and transforms it to distinct points in $z$, so that each point in $z$ is the image of some point in $\zeta$. This mapping would be one-to-one if distinct points in $\zeta$ are transformed to distinct points in $z$. In order that the mapping becomes conformal, it has to be one-to-one and has to preserve the magnitude and orientations of the angles between curves.

Let us consider the transformation $z=m(\zeta)$ which maps the region $R_{\zeta}$ in the imaginary $\zeta$-plane to the region $R_{z}$ in the real $z$-plane. Let $m(\zeta)$ be single valued so that specification of a certain $R_{\zeta}$ gives the corresponding $R_{z}$. Again let $m(\zeta)$ be the one-to-one and invertible so that we can get the region $R_{\zeta}$ if we know $R_{z}$ from the equation, $\zeta=m^{-1}(z)$. To ensure that the basic complex variable formulation is valid, let us also assume that $m(\zeta)$ is homomorphic in the region $R_{\zeta}$ and $m^{\prime}(\zeta) \neq 0$ at all points.

Suppose $L_{\zeta}$ is an arc passing through $\zeta_{0}$ in the region $R_{\zeta}$, then as $\zeta$ describes $L_{\zeta}$, the corresponding point $z$ will describe an arc $L_{z}$ in the region $R_{z}$ passing through the point $z_{0}=m\left(\zeta_{0}\right)$.

The relation between a small increment $\delta \zeta$ along $L_{\zeta}$ at $\zeta_{0}$ and the corresponding increment $\delta z$ along $L_{z}$ is $\delta z=m^{\prime}\left(\zeta_{0}\right) \delta \zeta$ (since m' $(\zeta)$ exists at all points in $R_{\zeta}$ ). From this, since $m^{\prime}\left(\zeta_{0}\right) \neq 0$, the mapping is locally one-to-one and invertible, the inverse relation being

$$
\delta \zeta=\delta z / m^{\prime}\left(\zeta_{0}\right)
$$

Again, since $|\mathrm{d} z / \mathrm{d} \zeta|=\left|m^{\prime}\left(\zeta_{0}\right)\right|$, the local magnification is $\left|m^{\prime}\left(\zeta_{0}\right)\right|$ and is independent of the direction of $L_{\zeta}$ at $\zeta=\zeta_{0}$.

Also,

$$
\arg [\delta z]=\arg \left[m^{\prime}\left(\zeta_{0}\right)\right]+\arg [\delta \zeta]
$$

Hence, the neighbourhood of the point $\zeta_{0}$ in the $\zeta$-plane is transformed by means of a magnification $\left|m^{\prime}\left(\zeta_{0}\right)\right|$ and a rigid body rotation through the angle $\arg \left[m^{\prime}\left(\zeta_{0}\right)\right]$ into the neighbourhood of the point $z_{0}$ in the $z$-plane provided $m^{\prime}\left(\zeta_{0}\right) \neq 0$. 
So if two $\operatorname{arcs} L_{\zeta}^{1}, L_{\zeta}^{2}$ intersect at $\zeta=\zeta_{0}$ with an angle of intersection $\phi$, then the corresponding $\operatorname{arcs} L_{\zeta}^{1}, L_{\zeta}^{2}$ in $R_{z}$ meet at $z_{0}$ in the same angle $\phi$ and with the same orientation, which means that the mapping becomes conformal.

Thus, the mapping becomes conformal when $m(\zeta)$ is holomorphic in $R_{\zeta}$ and $m^{\prime}(\zeta) \neq 0$ at all points in $R_{\zeta}$. These conditions also ensure that the inverse transformtion is conformal. It can be shown that conformal mapping defines a natural coordinate system in the mapped region $R_{z}$. This theory is dealt with in more detail in many textbooks (Churchill 1960; Carrier et al 1966). Kober (1952) has compiled a dictionary of conformal representations.

\subsection{Conformal mapping for plane linear elasticity}

Conformal mappings are widely used in plane linear elasticity because they help in transforming very complicated shapes into much simpler ones and allow the basic complex variable formulation to extend to the transformed problem, thereby making the powerful methods of solutions developed for circular and half-plane regions to be applicable to these problems. While solving boundary value problems, though conformal mapping gives more complex boundary conditions, it is preferred because of the simple shapes it gives. This method has been followed by Savin (1962) and others. Recently, Vandembroucq \& Roux (1997) have studied the properties of harmonic and biharmonic fields along rough edges of a semi-infinite boundary under uniaxial tension. Boundary stress perturbations caused by roughness are highlighted through Fourier statistics. Complex variables in conjunction with conformal mapping are employed by them to understand the role of surface roughness on plane boundaries. In this paper, boundary stresses around an irregular hole in a large stressed plate are obtained using complex variables in conjunction with conformal mapping. Unlike the papers by Vandembroucq \& Roux (1997), this paper provides exact results for the tangential stress $\sigma_{t}$ along the boundary of irregular holes for prescribed shapes. This prescription is achieved by a Laurent series form for conformally mapping the irregular hole onto a unit circle instead of a Fourier series formulation employed by Vandembroucq \& Roux (1997). Consequently, sharp corners in the form of cusps can be directly handled using only a few terms in the present approach. Thus, the results presented in this paper complement the work of Vandembroucq \& Roux (1997) to understand the physics and mechanics of roughness-induced phenomena. Handling roughness effects through numerical methods is generally inconvenient owing to many different scales simultaneously affecting the analysis. Such large variations pose overwhelming computational problems; and, it is difficult to interpret the numerical results. In this context, complex variables provide many useful and important information. Complex variables have been widely used in the theory of elasticity to tackle problems with well-defined geometry, but not for irregular complicated shapes such as those considered in this paper.

\subsection{Mapping procedure}

Here, we will be concerned with regions surrounding irregular holes which map conformally onto the exterior of the circle $|\zeta|=a$ in the complex plane. It can be shown that the infinite plane containing a single hole bounded by a contour $C$ can be mapped onto the region $|\zeta| \geq a$ (outside of a circle of radius $a$ ) in such a way that $m^{\prime}(\zeta) \neq 0$ for $|\zeta| \geq a$ if 
$\mathrm{C}$ has a continuous curvature. This mapping equation is given by

$$
z=m(\zeta)=a_{1} \zeta+a_{0}+\frac{b_{1}}{\zeta}+\frac{b_{2}}{\zeta^{2}}+\cdots+\frac{b_{n}}{\zeta^{n}}+\cdots
$$

Here it can be verified that $m(\zeta)$ is holomorphic in $R_{\zeta}$.

We can now, by a suitable choice of the axes, arrange that $a_{0}=0$ and $a_{1}=$ real to give

$$
z=m(\zeta)=R \zeta+\frac{b_{1}}{\zeta}+\frac{b_{2}}{\zeta^{2}}+\cdots+\frac{b_{n}}{\zeta^{n}}+\cdots
$$

Truncating the above series to $n$ terms provides an approximation to the mapping function and gives a region $R_{z}^{n}$ which is nearly of the same shape as the original region $R_{z}$. It can be shown that the solution of boundary value problems in elasticity for the region $R_{z}$ tends to the correct solution for the region $R_{z}$ as $n \rightarrow \infty$. So we have to take a value of $n$ which will give a good approximation to the actual problem. Here, we have truncated the series up to 9 negative power terms. Hence,

$$
z=m(\zeta)=R \zeta+\frac{b_{1}}{\zeta}+\cdots+\frac{b_{9}}{\zeta^{9}}
$$

Usually it is convenient to map onto the exterior of the unit circle $|\zeta| \geq 1$ and this procedure is adopted here. From (3), we can write

$$
z=m(\zeta)=R\left(\zeta+\frac{m_{1}}{\zeta}+\cdots+\frac{m_{9}}{\zeta^{9}}\right) .
$$

Since $R$ affects only the size and not the shape of the curve (hence curvature), it is not going to affect the stress distribution. Hence we can take $R=1$. Thus,

$$
z=m(\zeta)=\zeta+\frac{m_{1}}{\zeta}+\cdots+\frac{m_{9}}{\zeta^{9}}
$$

\subsection{Examples}

(1) The exterior of a unit circle can be mapped onto the exterior of an ellipse by the mapping equation

$$
z=m(\zeta)=\zeta+\frac{m_{1}}{\zeta}
$$

where $\left|m_{1}\right|<1$ to ensure that $m^{\prime}(\zeta) \neq 0$ for $|\zeta| \geq 1$ (figure 1 ).

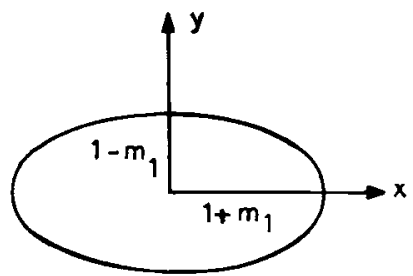

(a)

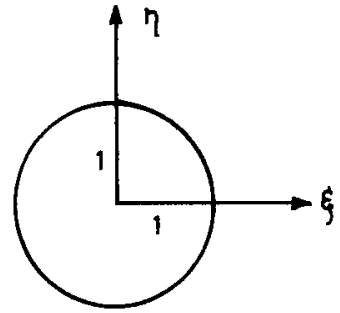

(b)

Figure 1. Conformal mapping. (a) z-plane ellipse, (b) complex plane circle. 

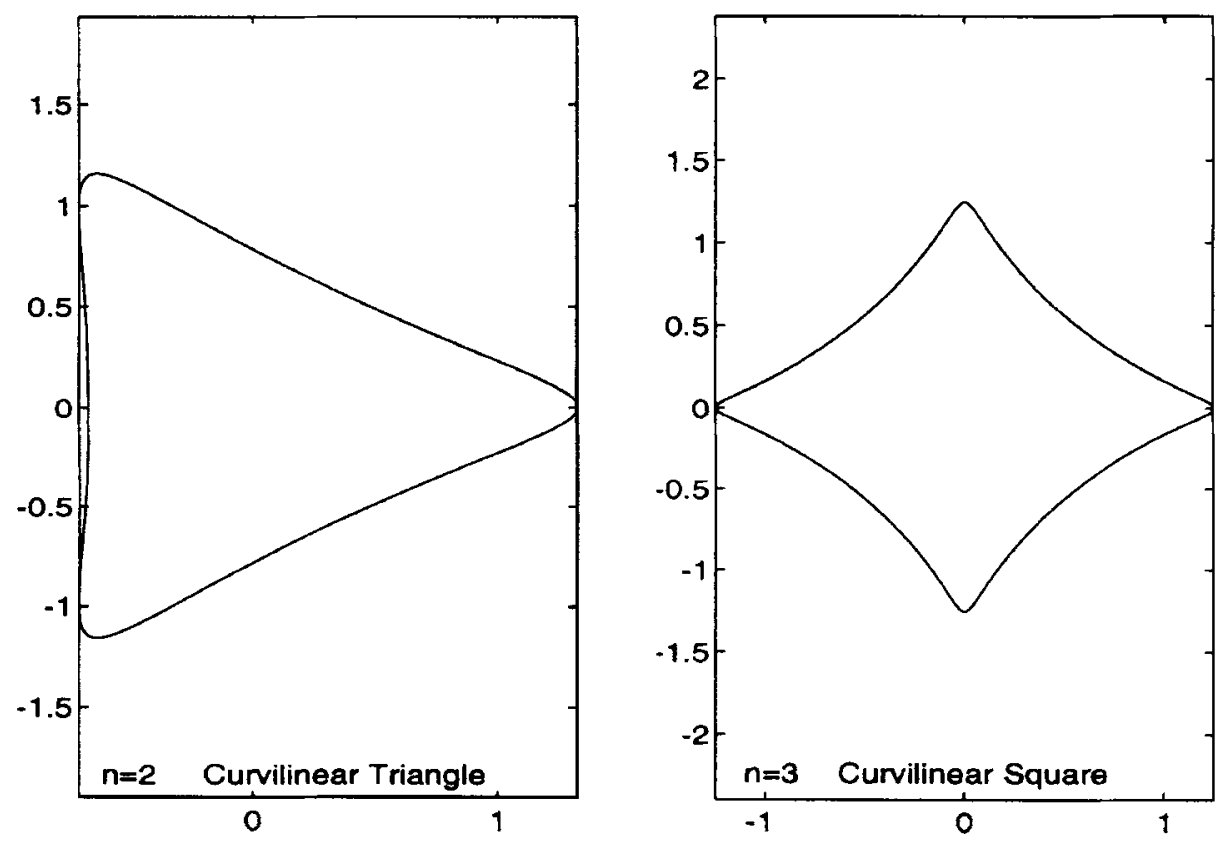

Figure 2. Hypotrochoidal shapes.
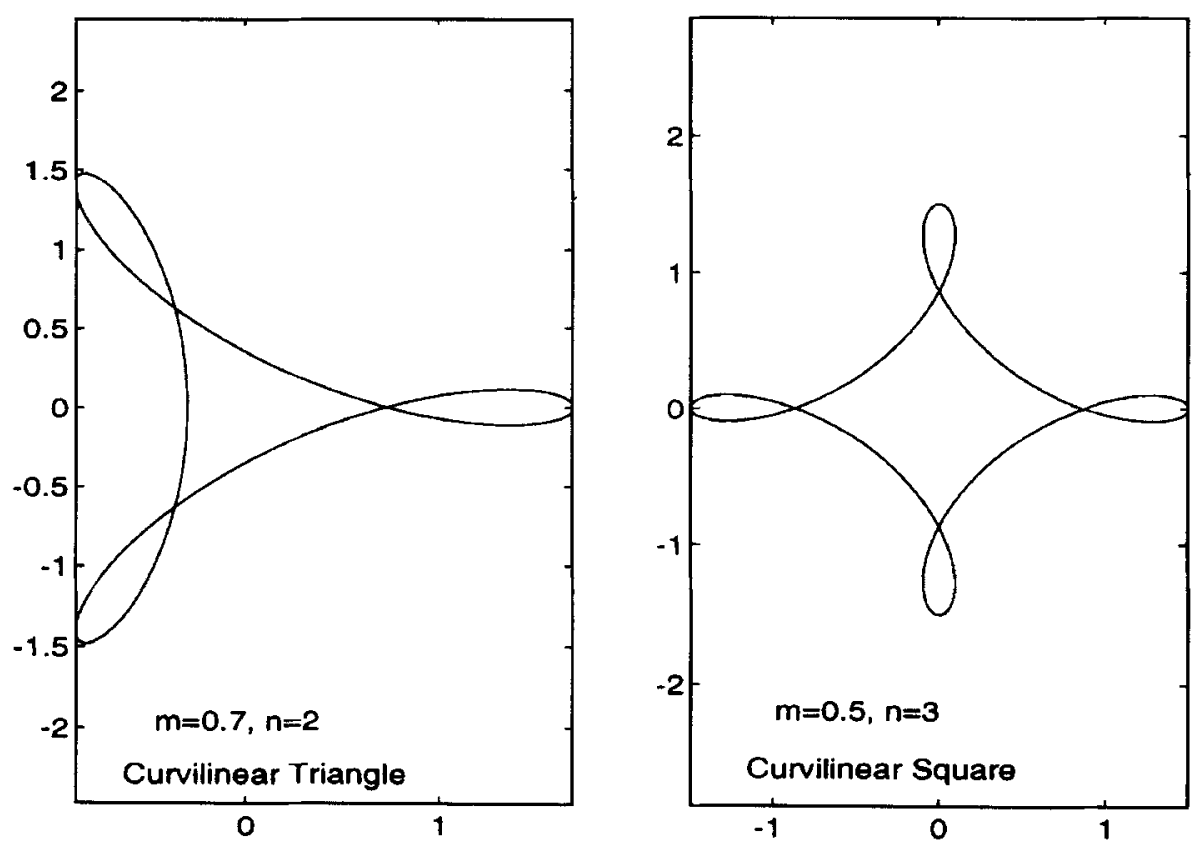

Figure 3. Looping in hypotrochoids. 
Since $\zeta=e^{i \theta}$ for a unit circle, $z=e^{i \theta}+m_{1} e^{-i \theta}$,

$$
\begin{aligned}
& x=\left(1+m_{1}\right) \cos \theta, \\
& y=\left(1-m_{1}\right) \sin \theta .
\end{aligned}
$$

The ellipse has semi-axes $\left(1+m_{1}\right)$ and $\left(1-m_{1}\right)$. In the two limiting cases, when $m_{1}=0$, the ellipse becomes a unit circle and when $m_{1}=1$, it becomes a crack of length $=4$ units.

(2) Hypotrochoids are loci of a point fixed relative to a moving circle which rolls without slipping upon the exterior of a fixed circle. The exterior of a unit circle can be mapped

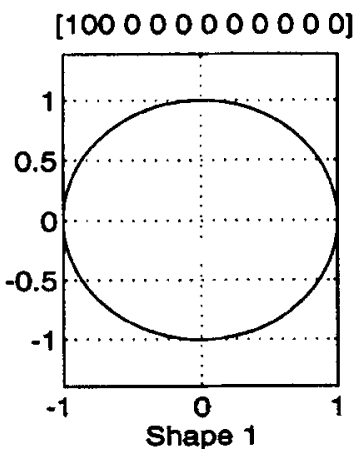

$[10000-150000-700]$

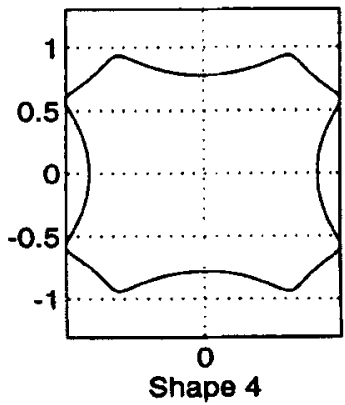

$[10050100000000]$

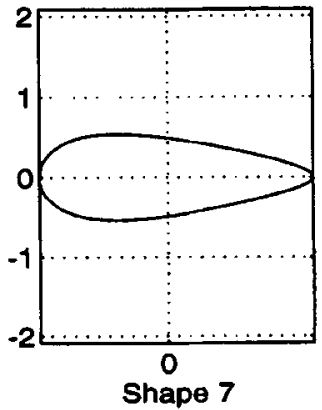

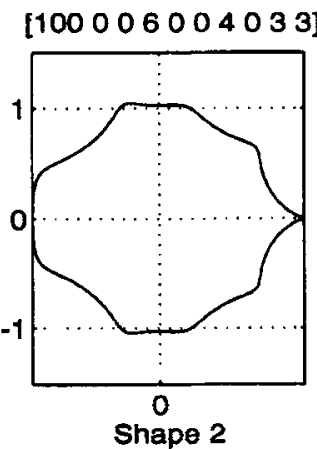

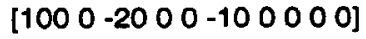

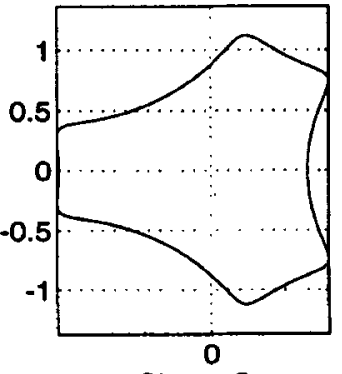

Shape 5

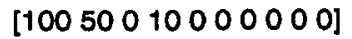

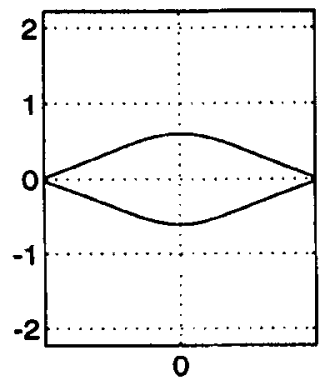

Shape 8

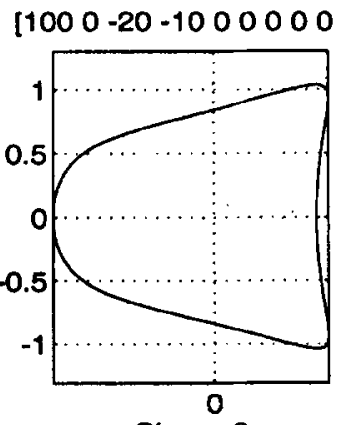

Shape 3

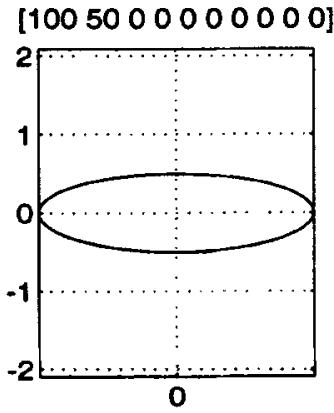

Shape 6

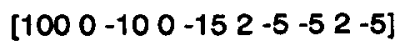

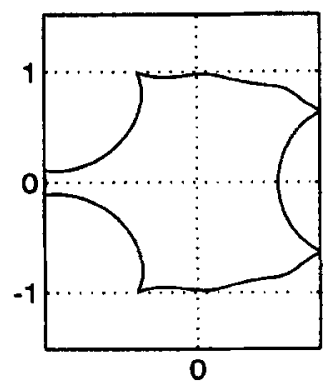

Shape 9

Figure 4. Selected shapes. 
Table 1. The shapes in ascending order of perimeter.

\begin{tabular}{|c|c|}
\hline Classification & Perimeter \\
\hline 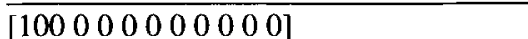 & 6.2832 \\
\hline 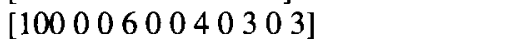 & 6.7640 \\
\hline$\left[\begin{array}{lllllllllll}100 & 0 & -20-10 & 0 & 0 & 0 & 0 & 0 & 0\end{array}\right]$ & 7.0870 \\
\hline 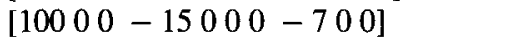 & 7.3030 \\
\hline$[1000-20000-1000000]$ & 7.3794 \\
\hline 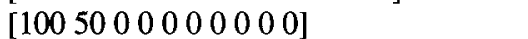 & 7.7164 \\
\hline$\left[\begin{array}{llllllllll}10 & 50 & 10 & 0 & 0 & 0 & 0 & 0 & 0 & 0\end{array}\right]$ & 7.9018 \\
\hline 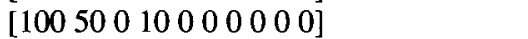 & 8.1352 \\
\hline$[1000-100-152-5-52-5]$ & 8.2134 \\
\hline
\end{tabular}

onto the exterior of a hypotrochoid by

$$
z=m(\zeta)=\zeta+\left(m_{n} / \zeta^{n}\right)
$$

where $n$ is a positive integer.

Putting $\zeta=e^{i \theta}$, we get the parametric equation of the hypotrochoid as

$$
z=e^{i \theta}+m_{n} e^{-i n \theta}
$$

The condition $0 \leq m_{n}<(1 / n)$ ensures that the hypotrochoid does not have loops (hypotrochoids are loci of a point fixed relative to a moving circle which rolls without slipping upon the exterior of a fixed circle).

For $n=2$, the mapping gives a curvilinear triangle and for $n=3$, a curvilinear square (figure 2). Loops result when $m>\frac{1}{2}$ for the triangle and when $m>\frac{1}{3}$ for curvilinear square as shown (figure 3).

(3) Taking terms up to $m_{9}$, we can get different complex shapes which are symmetric about the $x$-axis. Here $m$ 's can be positive or negative and we can have $9 C_{1}+9 C_{2}+$ $9 C_{3}+9 C_{4}+9 C_{5}+9 C_{6}+9 C_{7}+9 C_{8}+9 C_{9}=1022$ number of combinations. Some typical shapes are considered in figure 4 . The corresponding values of $m$ are listed in table 1 in increasing order of perimeter. The first shape considered is a circle of unit radius. According to this order of increasing perimeter but fixed area, an ellipse is the 6th shape. Other shapes considered are quite complicated, but the powerful technique of conformal mapping renders the problem tractable.

The code which has been followed here for a curve,

$$
z=\zeta+\frac{m_{1}}{\zeta}+\cdots+\frac{m_{9}}{\zeta^{9}},
$$

is that the curve has been indicated by the code $\left[100,100 m_{1}, 100 m_{2}, \ldots, 100 m_{9}\right]$.

For example, the code $[100,30,0,0,0,0,0,0,0,3]$ indicates

$$
z=\zeta+\frac{0.3}{\zeta}+\frac{0.03}{\zeta^{9}} \text {. }
$$

\section{Hydrostatic loading}

We will be concerned in this section with hydrostatic tension of a large elastic plate with a small irregular hole. This is a special case of biaxial loading with equal principal stresses 
$\left(\sigma_{1}=\sigma_{2}\right)$. Since there is no traction applied at the boundary of the inner hole (free edge), the normal stress is zero at the points along the hole. A large number of practical problems fall in this category. Spherical pressure vessels, the centre of a rotating disk, and the state of stress on the crack line under mode 1 loading, are some examples. The presence of irregular cavities or microvoids induce large stress concentrations.

\subsection{Curvilinear regular polygonal holes}

As discussed in the previous chapter, the conformal mapping for curvilinear regular polygons or hypotrochoids is $z=\zeta+\left(m_{n} / \zeta^{n}\right)$ where $n$ is a positive integer.

Since here only one $m$ term exists, we drop the subscript for convenience.

As discussed previously, the condition for non-looping of the curve is $m n<1$.

Now the tangential stress distribution around this shaped hole for unit hydrostatic tension applied remotely is well known and given by (Givoli \& Elishakoff 1992)

$$
\sigma_{t}=\frac{2\left(1-m^{2} n^{2}\right)}{1+m^{2} n^{2}-2 m n \cos (n+1) \theta},
$$

where $\theta=$ angle in the $\zeta$-plane; and $\zeta=e^{i \theta}=\cos \theta+i \sin \theta$.

We can get results for special cases from this general equation.

For the case of the ellipse, $n=1$ and $m$ can be obtained in terms of the semi axes $a, b$ as follows

$$
m=(a-b) /(a+b) .
$$

Let the ratio of axes be $3: 1$ i.e., $a=3 b(m=1 / 2)$. Table 2 gives the $\sigma_{t}$ variation for a $3: 1$ ellipse based on (5) as $6 /(5-4 \cos 2 \theta)$.

\subsection{General formula for arbitrary holes}

By taking more terms in the conformal mapping equation, we can get different curves, which can be of any shape but are symmetric about the $x$-axis. The analysis for stress distribution around these holes will be done here.

The sum of the orthogonal stresses $\left(\sigma_{x}+\sigma_{y}\right)$ or $\left(\sigma_{t}+\sigma_{n}\right)$ at any point of the elastic body under equilibrium is given by

$$
\sigma_{x}+\sigma_{y}=\sigma_{t}+\sigma_{n}=4 \operatorname{Re}(\mathrm{d} E / \mathrm{d} z) .
$$

Table 2. Tangential stress around the ellipse.

\begin{tabular}{lc}
\hline Angle $\theta$ in & Tangential stress \\
complex plane & $\sigma_{t}$ \\
\hline 0 & 6 \\
$\pi / 6$ & 2 \\
$\pi / 4$ & 1.2 \\
$\pi / 2$ & $2 / 3$ \\
\hline
\end{tabular}


Now, the first complex potential corresponding to a hypotrochoidal hole $(z=\zeta+$ $\left.\left(m / \zeta^{n}\right),|\zeta| \geq 1\right)$ in an infinite solid inflated by a uniform pressure $p$ with zero stress and rotation at infinity is (England 1971)

$$
E=-p m / \zeta^{n}
$$

For a hypotrochoidal hole,

$$
\sigma_{t}+\sigma_{n}=4 \operatorname{Re} \frac{\mathrm{d} E}{\mathrm{~d} z}=4 \operatorname{Re}\left(\frac{\mathrm{d} E}{\mathrm{~d} \zeta} / \frac{\mathrm{d} z}{\mathrm{~d} \zeta}\right)=4 \operatorname{Re} \frac{(p m n) /\left(\zeta^{n+1}\right)}{1-\left(m n / \zeta^{n+1}\right)}
$$

or

$$
\frac{\sigma_{t}+\sigma_{n}}{4 p}=\operatorname{Re} \frac{(m n) /\left(\zeta^{n+1}\right)}{1-\left(m n / \zeta^{n+1}\right)}
$$

Now for an irregular hole, $z=\zeta+\sum\left(m_{n} / \zeta^{n}\right), E$ will be $\sum-p\left(m_{n} / \zeta^{n}\right)$. Thus for this case, $(\mathrm{d} E / \mathrm{d} \zeta)=p \sum\left(n m_{n} / \zeta^{n+1}\right)=p(1-(\mathrm{d} z / \mathrm{d} \zeta))$ since, $(\mathrm{d} z / \mathrm{d} \zeta)=m(\zeta)=$ $1-\sum\left(n m_{n} / \zeta^{n+1}\right)$. Therefore,

$$
\frac{\sigma_{t}+\sigma_{n}}{4 p}=\operatorname{Re} \frac{1-m^{\prime}(\zeta)}{m^{\prime}(\zeta)}
$$

To derive the result under hydrostatic tension (HT), we use superposition.

$$
\left(\sigma_{t}+\sigma_{n}\right)_{\mathrm{HT}}=\left(\sigma_{t}+\sigma_{n}\right)_{\mathrm{IP}}+2 p,
$$

where the suffix IP denotes internal pressure. For unit internal pressure, $p=1$. Hence

$$
\left(\sigma_{t}+\sigma_{n}\right)_{H T}=4 \operatorname{Re} \frac{1-m^{\prime}(\zeta)}{m^{\prime}(\zeta)}+2=4 \operatorname{Re} \frac{1}{m^{\prime}(\zeta)}-2
$$

Since there is no normal force on the hole boundary, $\sigma_{n}=0$. So,

$$
\sigma_{t}=4 \operatorname{Re} \frac{1}{m^{\prime}(\zeta)}-2
$$

In summary, the boundary stress variation around small irregular holes in a large plate under hydrostatic tension is determined directly from the conformal mapping equation.

\section{Uniaxial loading}

\subsection{General irregular hole}

The tangential stress distribution around a hole of any shape under uniaxial loading is given by

$$
\sigma_{t}=4 \operatorname{Re}\left(E^{\prime} / m^{\prime}\right)
$$

As an example, let

$$
z=m(\zeta)=\zeta+\frac{m_{3}}{\zeta^{3}}+\frac{m_{7}}{\zeta^{7}}
$$


and

$$
E=\frac{\sigma \zeta}{4}+\frac{\alpha_{1}}{\zeta}+\frac{\alpha_{2}}{\zeta^{2}}+\ldots+\frac{\alpha_{7}}{\zeta^{7}}
$$

To determine $\alpha_{1}, \alpha_{2}, \ldots, \alpha_{7}$ in $E$, we equate like powers of $\zeta$ (only for negative powers) in the following equation developed using complex analysis for the plane elastic problems (Timoshenko \& Goodier 1970; England 1971).

$$
E_{0}-\zeta^{2} M\left(\overline{\alpha_{1}}+2 \overline{\alpha_{2}} \zeta+\cdots+7 \overline{\alpha_{7}} \zeta^{6}\right)=\frac{\sigma e^{2 i \alpha_{L}}}{2 \zeta}-\frac{\sigma M}{4},
$$

where $E_{0}=\left(\alpha_{1} / \zeta\right)+\left(\alpha_{2} / \zeta^{2}\right)+\cdots+\left(\alpha_{7} / \zeta^{7}\right) ; M=\left(m_{3} / \zeta^{3}\right)+\left(m_{7} / \zeta^{7}\right)$ and $\alpha_{L}$ is the loading direction with respect to the $x$-axis. Substituting for $E_{0}$ and $M$,

$$
\begin{aligned}
\frac{\alpha_{1}}{\zeta} & +\frac{\alpha_{2}}{\zeta^{2}}+\cdots+\frac{\alpha_{7}}{\zeta^{7}}-\left(\frac{m_{3}}{\zeta}+\frac{m_{7}}{\zeta^{5}}\right)\left(\overline{\alpha_{1}}+2 \overline{\alpha_{2}} \zeta+3 \overline{\alpha_{3}} \zeta^{2}+\cdots+7 \overline{\alpha_{7}} \zeta^{6}\right) \\
& =\frac{\sigma e^{2 i \alpha_{L}}}{2 \zeta}-\left[\sigma\left(\frac{m_{3}}{\zeta}+\frac{m_{7}}{\zeta^{5}}\right) / 4\right] .
\end{aligned}
$$

Now equating coefficients of like powers of $\zeta$ (only for negative powers), we get

$$
\begin{array}{lll}
\zeta^{-1}: \alpha_{1}-\overline{\alpha_{1}} m_{3}-5 \overline{\alpha_{5}} m_{7} & =\sigma e^{2 i \alpha} L / 2 \\
\zeta^{-2}: \alpha_{2}-4 \overline{\alpha_{4}} m_{7} & =0, \\
\zeta^{-3}: \alpha_{3}-3 \overline{\alpha_{3}} m_{7} & =-\sigma m_{3} / 4 \\
\zeta^{-4}: \alpha_{4}-2 \overline{\alpha_{2}} m_{7} & =0, \\
\zeta^{-5}: \alpha_{5}-\overline{\alpha_{1}} m_{7} & =0 \\
\zeta^{-6}: \alpha_{6} & =0 \\
\zeta^{-7}: \alpha_{7} & =-\sigma m_{7} / 4 .
\end{array}
$$

Thus,

$$
\begin{aligned}
& \alpha_{6}=\alpha_{4}=\alpha_{2}=0 ; \\
& \alpha_{7}=\frac{\sigma m_{7}}{4} ; \\
& \alpha_{5}=\overline{\alpha_{1} m_{7}, \overline{\alpha_{5}}=\alpha m_{7} ;} \\
& \alpha_{3}=\overline{\alpha_{3}}=-\frac{\sigma m_{3}}{4\left(1-3 m_{7}\right)} .
\end{aligned}
$$

From the $\zeta^{-1}$ equation,

$$
\alpha_{1}\left(1-5 m_{7}^{2}\right)-\overline{\alpha_{1}} m_{3}=\frac{1}{2} \sigma e^{2 i \alpha} L .
$$

Equating real and imaginary parts of $\alpha_{1}=\alpha_{1 R}+i \alpha_{1 C}$,

$$
\begin{gathered}
\alpha_{1 R}\left(1-5 m_{7}^{2}-m_{3}\right)=\frac{1}{2} \sigma \cos 2 \alpha_{L}, \\
\alpha_{1 C}\left(1-5 m_{7}^{2}+m_{3}\right)=\frac{1}{2} \sigma \sin 2 \alpha_{L} .
\end{gathered}
$$

Knowing $\alpha_{1}, \alpha_{2}, \ldots, \alpha_{7}, \sigma_{t}$ is determined. 


\subsection{Special cases}

4.2a $\quad$ Ellipse: $\quad z=m(\zeta)=\zeta+\left(m_{1} / \zeta\right)$

Here

$$
E=(\sigma \zeta / 4)+\left(\alpha_{1} / \zeta\right)
$$

Hence,

$$
E_{0}-\zeta^{2} M\left(\overline{\alpha_{1}}\right)=\left(\sigma e^{2 i \alpha_{L}} / 2 \zeta\right)-(\sigma M / 4),
$$

where $E_{0}=\left(\alpha_{1} / \zeta\right)$ and $M=\left(m_{1} / \zeta\right)$.

Hence,

$$
\frac{\alpha_{1}}{\zeta}-\zeta^{2} \frac{m_{1}}{\zeta} \overline{\alpha_{1}}=\frac{\sigma e^{2 i \alpha_{L}}}{2 \zeta}-\frac{\sigma m_{1}}{4 \zeta}
$$

or

$$
\begin{aligned}
\alpha_{1} & =\frac{\sigma e^{2 i \alpha_{L}}}{2}-\frac{\sigma m_{1}}{4} \\
E & =\frac{\sigma \zeta}{4}+\frac{\sigma e^{2 i \alpha_{L}}}{2 \zeta}-\frac{\sigma m_{1}}{4 \zeta} \\
E^{\prime} & =\frac{\sigma e^{2 i \alpha_{L}}}{2 \zeta^{2}}+\frac{\sigma m_{1}}{4 \zeta^{2}}=\frac{\sigma}{4}+\frac{\sigma}{4}\left(m_{1}-2 e^{2 i \alpha_{L}}\right) \zeta^{-2}
\end{aligned}
$$

Therefore,

$$
\sigma_{t}=4 \operatorname{Re}\left[\frac{\sigma}{4}+\frac{\sigma}{4}\left(m_{1}-2 e^{2 i \alpha_{L}}\right) \zeta^{-2} /\left(1-\frac{m_{1}}{\zeta^{2}}\right)\right] .
$$

This agrees with that given by England (1971).

4.2b Curvilinear square: $\quad z=\zeta+\left(m_{3} / \zeta^{3}\right)$.

Here

$$
E=\frac{\sigma \zeta}{4}+\frac{\alpha_{1}}{\zeta}+\frac{\alpha_{2}}{\zeta^{2}}+\frac{\alpha_{3}}{\zeta^{3}}
$$

Let $m_{3}=1 / 4$. To get $\alpha_{1}, \alpha_{2}, \alpha_{3}$ we equate

$$
E_{0}-\zeta^{2} M\left(\overline{\alpha_{1}}+2 \overline{\alpha_{2}} \zeta+3 \overline{\alpha_{3}} \zeta^{2}\right)=\frac{\sigma e^{2 i \alpha_{L}}}{2 \zeta}-\frac{\sigma M}{4},
$$

where $E_{0}=\left(\alpha_{1} / \zeta\right)+\left(\alpha_{2} / \zeta^{2}\right)+\left(\alpha_{3} / \zeta^{3}\right)$ and $\left(M=m_{3} / \zeta^{3}\right)=\left(1 / 4 \zeta^{3}\right)$.

Equating the coefficients of like powers of $\zeta$, we get

$$
\begin{aligned}
& \alpha_{1}-m_{3} \bar{\alpha}_{1}=\frac{1}{2} \sigma e^{2 i \alpha_{L}}, \\
& \alpha_{2}=0, \\
& \alpha_{3}=-\sigma / 16,
\end{aligned}
$$


Table 3. Tangential stress around curvilinear square.

\begin{tabular}{lcccc}
\hline Point & $\theta$ & $E^{\prime}$ & $m^{\prime}$ & $\sigma_{t}$ \\
\hline A & 0 & $-\frac{11}{48}$ & $\frac{1}{4}$ & $-\frac{11}{3}$ \\
B & $\pi / 4$ & $\frac{1}{16}+\frac{2}{3} i$ & $\frac{7}{4}$ & $\frac{1}{7}$ \\
C & $\pi / 2$ & $\frac{53}{48}$ & $\frac{1}{4}$ & $\frac{53}{3}$ \\
\hline
\end{tabular}

Suppose, a unit uniaxial load is applied at an angle $0^{\circ}$, i.e., $\sigma=1$ and $\alpha_{L}=0$. Then $\alpha_{1}=2 / 3 ; \alpha_{2}=0 ; \alpha_{3}=-1 / 16$ and $E=(\zeta / 4)+(2 / 3 \zeta)-\left(1 / 16 \zeta^{3}\right)$.

Since $m^{\prime}=1-\left(3 / 4 \zeta^{4}\right)$, we can get the values for the $\sigma_{t}$ variation as in table 3 . If we apply load at $90^{\circ}$ to the $x$-axis, the total tangential stress at $\mathrm{A}, \mathrm{B}, \mathrm{C}$ due to the two forces are

$$
\begin{array}{ll}
A: & \sigma_{t}=-\frac{11}{3}+\frac{53}{3}=14 \\
B: & \sigma_{t}=\frac{1}{7}+\frac{1}{7}=\frac{2}{7} \\
C: & \sigma_{t}=\frac{53}{3}-\frac{11}{3}=14 .
\end{array}
$$

The above result can also be obtained directly using the results from the previous section which is

$$
\sigma_{t}=4 \operatorname{Re}\left(1 / m^{\prime}\right)-2 .
$$
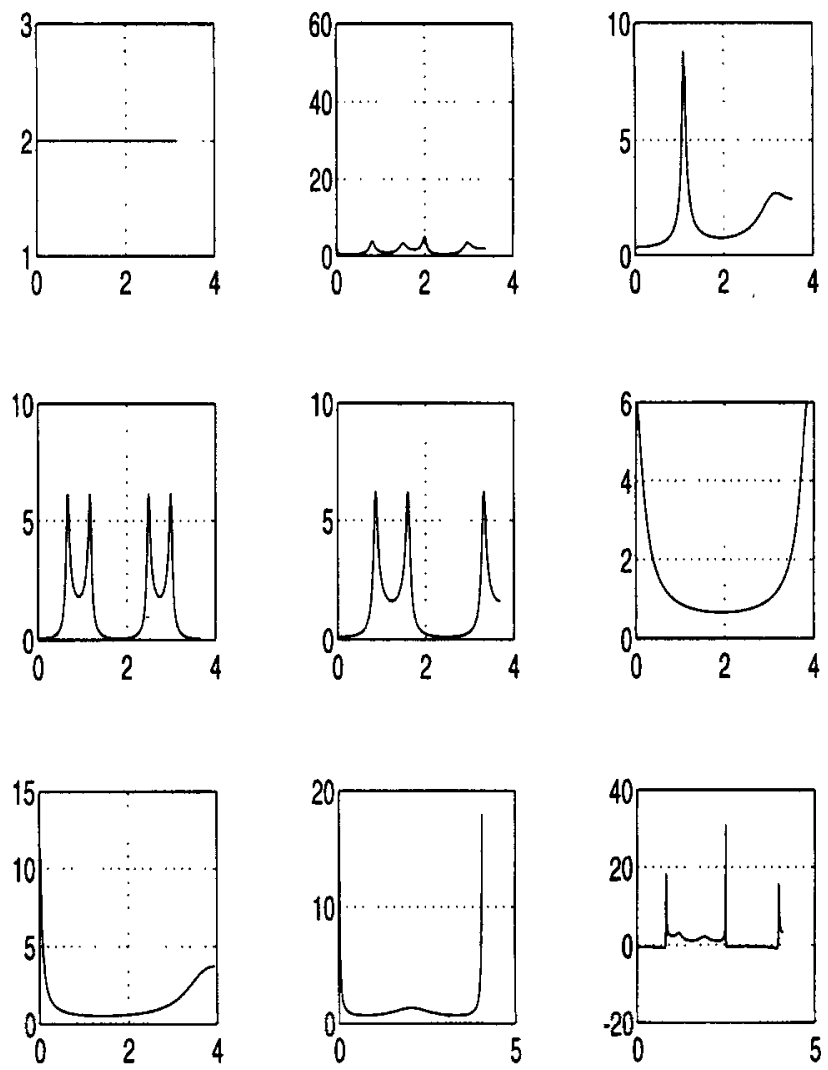

Figure 5. Stress distribution for hydrostatic loading. 
Hence,

$$
\begin{array}{ll}
\text { at } A, \theta=0^{\circ}, & \sigma_{t}=4\left(1 / \frac{1}{4}\right)-2=14, \\
\text { at } B, \theta=45^{\circ}, & \sigma_{t}=4\left(1 / \frac{7}{4}\right)-2=\frac{2}{7}, \\
\text { at } C, \theta=90^{\circ}, & \sigma_{t}=4\left(1 / \frac{1}{4}\right)-2=14 .
\end{array}
$$

\subsection{General case with $9 m$ terms}

Let the hole be given by the conformal mapping

$$
z=m(\zeta)=\zeta+\left(m_{1} / \zeta\right)+\cdots+\left(m_{9} / \zeta_{9}\right) .
$$

The complex potential $E$ is

$$
E=\frac{\sigma \zeta}{4}+\frac{\alpha_{1}}{\zeta}+\frac{\alpha_{2}}{\zeta^{2}}+\cdots+\frac{\alpha_{9}}{\zeta^{9}}
$$

So,

$$
E_{0}-\zeta^{2} M\left(\overline{\alpha_{1}}+2 \overline{\alpha_{2}} \zeta+3 \overline{\alpha_{3}} \zeta^{2}+\cdots+9 \overline{\alpha_{9}} \zeta^{8}\right)=\frac{\sigma e^{2 i \alpha_{L}}}{2 \zeta}-\frac{\sigma M}{4},
$$

where $E_{0}=\left(\alpha_{1} / \zeta\right)+\left(\alpha_{2} / \zeta^{2}\right)+\cdots+\left(\alpha_{7} / \zeta^{7}\right)$ and $M=\left(m_{1} / \zeta\right)+\cdots+\left(m_{9} / \zeta^{9}\right)$.

Substituting for $E_{0}$ and $M$,

$$
\begin{aligned}
& \frac{\alpha_{1}}{\zeta}+\cdots+\frac{\alpha_{9}}{\zeta^{9}}-\left(m_{1} \zeta+m_{2}+\frac{m_{3}}{\zeta}+\cdots+\frac{m_{9}}{\zeta^{7}}\right)\left(\overline{\alpha_{1}}+2 \overline{\alpha_{2}} \zeta+\cdots+9 \overline{\alpha_{9}} \zeta^{8}\right) \\
& =\frac{\sigma e^{2 i \alpha_{L}}}{2 \zeta}-\frac{\sigma}{4}\left(\frac{m_{1}}{\zeta}+\cdots+\frac{m_{9}}{\zeta^{9}}\right) .
\end{aligned}
$$

Equating the coefficients of like powers of $\zeta$ (only for negative powers),

$$
\begin{gathered}
{\left[\begin{array}{lcccccccc}
m_{3} & 2 m_{4} & 3 m_{5} & 4 m_{6} & 5 m_{7} & 6 m_{8} & 7 m_{9} & 0 & 0 \\
m_{4} & 2 m_{5} & 3 m_{6} & 4 m_{7} & 5 m_{8} & 6 m_{9} & 0 & 0 & 0 \\
m_{5} & 2 m_{6} & 3 m_{7} & 4 m_{8} & 5 m_{9} & 0 & 0 & 0 & 0 \\
m_{6} & 2 m_{7} & 3 m_{8} & 4 m_{9} & 0 & 0 & 0 & 0 & 0 \\
m_{7} & 2 m_{8} & 3 m_{9} & 0 & 0 & 0 & 0 & 0 & 0 \\
m_{8} & 2 m_{9} & 0 & 0 & 0 & 0 & 0 & 0 & 0 \\
m_{9} & 0 & 0 & 0 & 0 & 0 & 0 & 0 & 0 \\
0 & 0 & 0 & 0 & 0 & 0 & 0 & 0 & 0 \\
0 & 0 & 0 & 0 & 0 & 0 & 0 & 0
\end{array}\right]\left[\begin{array}{c}
\overline{\alpha_{1}} \\
\overline{\alpha_{2}} \\
\overline{\alpha_{3}} \\
\overline{\alpha_{4}} \\
\overline{\alpha_{5}} \\
\overline{\alpha_{6}} \\
\overline{\alpha_{7}} \\
\overline{\alpha_{8}} \\
\overline{\alpha_{9}}
\end{array}\right]} \\
=\left[\begin{array}{c}
\alpha_{1} \\
\alpha_{2} \\
\alpha_{3} \\
\alpha_{4} \\
\alpha_{5} \\
\alpha_{6} \\
\alpha_{7} \\
\alpha_{8} \\
\alpha_{9}
\end{array}\right]+\frac{\sigma}{4}\left[\begin{array}{c}
m_{1}-2 e^{2 i \alpha_{L}} \\
m_{2} \\
m_{3} \\
m_{4} \\
m_{5} \\
m_{6} \\
m_{7} \\
m_{8} \\
m_{9}
\end{array}\right] .
\end{gathered}
$$



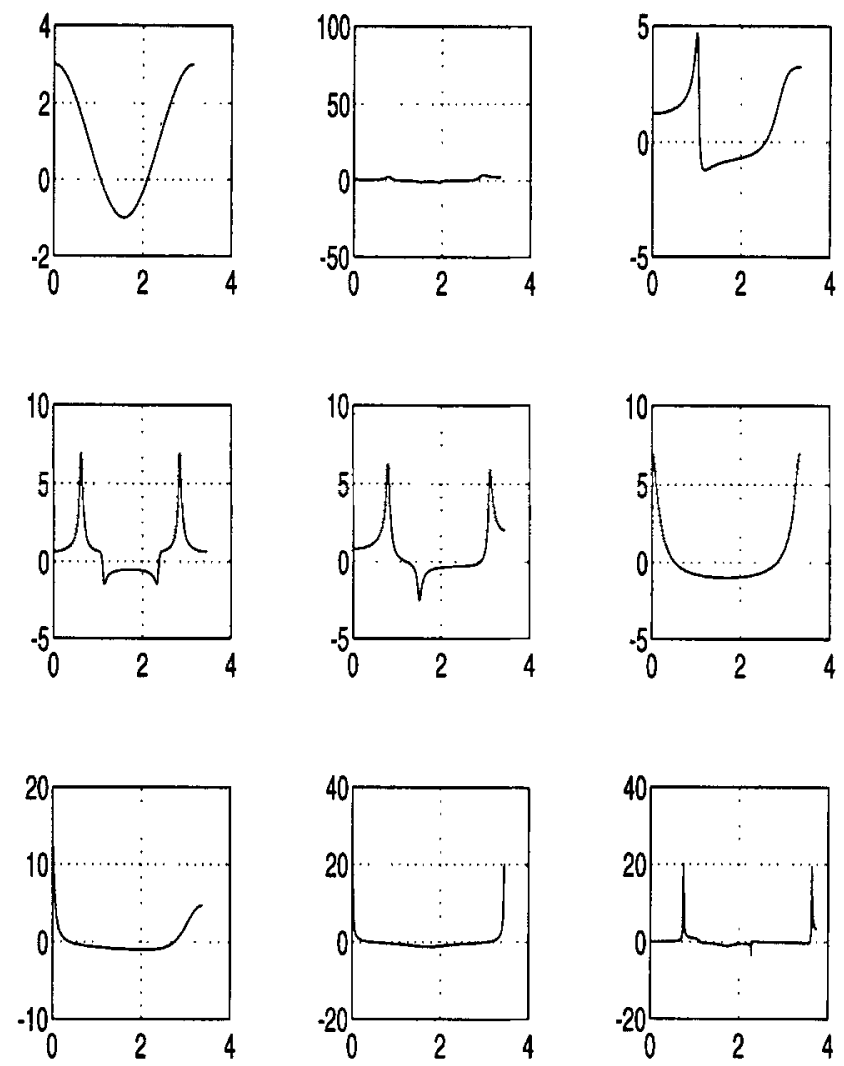

Figure 6. Stress distribution for uniaxial loading at 90 degrees.

Thus we can get $\alpha_{8}$ and $\alpha_{9}$ directly. Since $\alpha_{1}, \ldots, \alpha_{7}$ are complex, we have to resolve the equations into real and imaginary parts to get $[C][\alpha]=[U]$, where $C=\left[\left[C_{R}\right]\left[C_{C}\right]\right]$; and,

$$
C_{R}=\left(\begin{array}{ccccccc}
1-m_{3} & -2 m_{4} & -3 m_{5} & -4 m_{6} & -5 m_{7} & -6 m_{8} & -7 m_{9} \\
0 & 0 & 0 & 0 & 0 & 0 & 0 \\
-m_{4} & 1-2 m_{3} & -3 m_{6} & -4 m_{7} & -5 m_{8} & -6 m_{9} & 0 \\
0 & 0 & 0 & 0 & 0 & 0 & 0 \\
-m_{5} & -2 m_{6} & 1-3 m_{7} & -4 m_{8} & -5 m_{9} & 0 & 0 \\
0 & 0 & 0 & 0 & 0 & 0 & 0 \\
-m_{6} & -2 m_{7} & -3 m_{8} & 1-4 m_{9} & 0 & 0 & 0 \\
0 & 0 & 0 & 0 & 0 & 0 & 0 \\
-m_{7} & -2 m_{8} & -3 m_{9} & 0 & 1 & 0 & 0 \\
0 & 0 & 0 & 0 & 0 & 0 & 0 \\
-m_{8} & -2 m_{9} & 0 & 0 & 0 & 1 & 0 \\
0 & 0 & 0 & 0 & 0 & 0 & 0 \\
-m_{9} & 0 & 0 & 0 & 0 & 0 & 1 \\
0 & 0 & 0 & 0 & 0 & 0 & 0
\end{array}\right),
$$




$$
\begin{aligned}
& C_{C}=\left(\begin{array}{ccccccc}
0 & 0 & 0 & 0 & 0 & 0 & 0 \\
1+m_{3} & 2 m_{4} & 3 m_{5} & 4 m_{6} & 5 m_{7} & 6 m_{8} & 7 m_{9} \\
0 & 0 & 0 & 0 & 0 & 0 & 0 \\
m_{4} & 1+2 m_{5} & 3 m_{6} & 4 m_{7} & 5 m_{8} & 6 m_{9} & 0 \\
0 & 0 & 0 & 0 & 0 & 0 & 0 \\
m_{5} & 2 m_{6} & 1+3 m_{7} & 4 m_{8} & 5 m_{9} & 0 & 0 \\
0 & 0 & 0 & 0 & 0 & 0 & 0 \\
m_{6} & 2 m_{7} & 3 m_{8} & 1+4 m_{9} & 0 & 0 & 0 \\
0 & 0 & 0 & 0 & 0 & 0 & 0 \\
m_{7} & 2 m_{8} & 3 m_{9} & 0 & 1 & 0 & 0 \\
0 & 0 & 0 & 0 & 0 & 0 & 0 \\
m_{8} & 2 m_{9} & 0 & 0 & 0 & 1 & 0 \\
0 & 0 & 0 & 0 & 0 & 0 & 0 \\
m_{9} & 0 & 0 & 0 & 0 & 0 & 1
\end{array}\right), \\
& \alpha=\left(\begin{array}{c}
\alpha_{1 R} \\
\alpha_{2 R} \\
\alpha_{3 R} \\
\alpha_{4 R} \\
\alpha_{5 R} \\
\alpha_{6 R} \\
\alpha_{7 R} \\
\alpha_{1 C} \\
\alpha_{2 C} \\
\alpha_{3 C} \\
\alpha_{4 C} \\
\alpha_{5 C} \\
\alpha_{6 C} \\
\alpha_{7 C}
\end{array}\right) ; \quad U=\left(\begin{array}{c}
\frac{\sigma \cos 2 \alpha_{L}}{2}-\frac{\sigma m_{1}}{4} \\
\frac{\sigma \sin 2 \alpha_{L}}{2} \\
-\frac{\sigma m_{2}}{4} \\
0 \\
-\frac{\sigma m_{3}}{4} \\
0 \\
-\frac{\sigma m_{4}}{4} \\
0 \\
-\frac{\sigma m_{5}}{4} \\
0 \\
-\frac{\sigma m_{6}}{4} \\
0 \\
-\frac{\sigma m_{7}}{4} \\
0
\end{array}\right)
\end{aligned}
$$

We can solve this system of equations and get the matrix $\alpha$.

\section{Pure shear loading}

The pure shear state can be obtained by the superposition of equal and opposite stresses $\sigma$ and $-\sigma$ at $\alpha_{L}=(\pi / 4)$ and $\alpha_{L}=(3 \pi / 4)$, respectively.

For the two cases of loading, we will get different variations of tangential stress and we can superimpose the two variations to get the result for pure shear.

\section{Results and discussion}

Using the results obtained in the previous sections, the boundary stress variation is plotted in figures 5 to 9 for hydrostatic loading, uniaxial loading, and pure shear. The boundary 

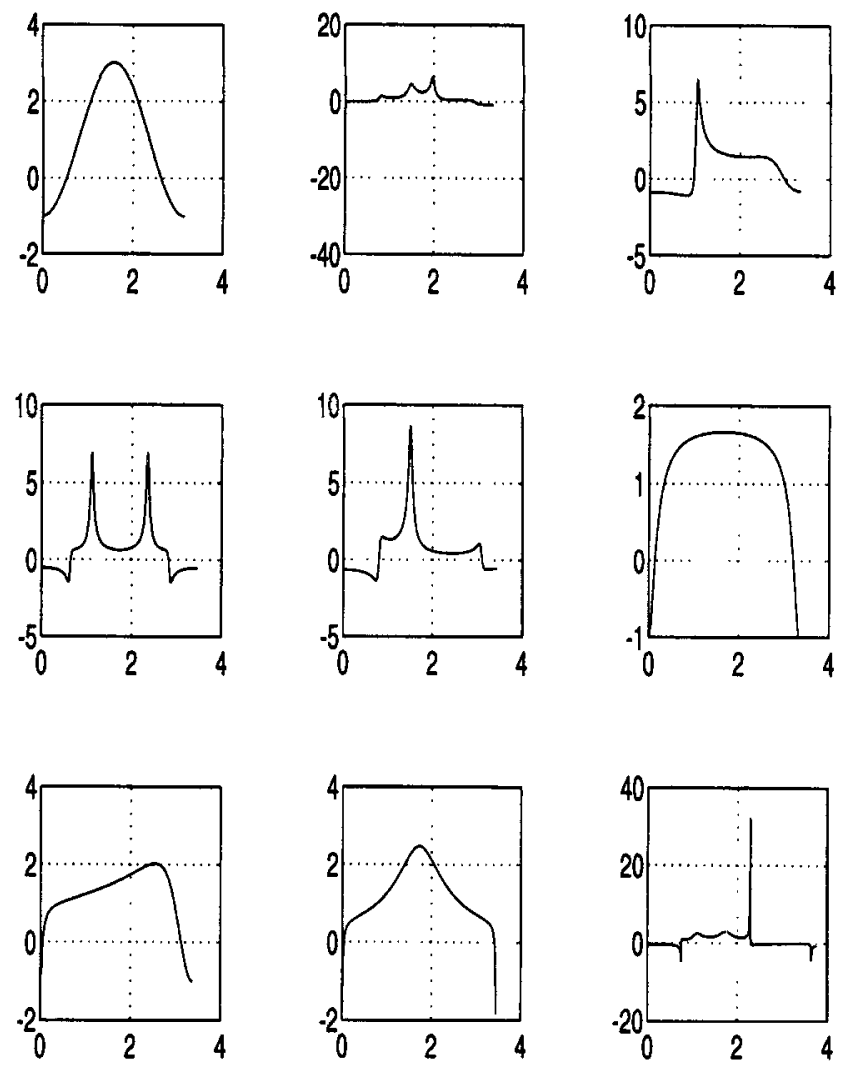

Figure 7. Stress distribution for uniaxial loading at 0 degrees.

stress is plotted as a function of the perimetric distance from $\theta=0$ in the counterclockwise direction. For hydrostatic loading, $\sigma_{t}$ is plotted only up to $\theta=\pi$ due to symmetry (figure 5). In figure 6, the loading angle $\alpha_{L}=\pi / 2$ and in figure 7, the loading angle is 0 . Again in the above two cases, the stress variation is symmetric about the $x$-axis. In a general case, for $\alpha_{L} \neq 0, \pi / 2$, the boundary stress variation does not exhibit symmetry as in figure 8 which shows the boundary stress variation for $\alpha_{L}=\pi / 6$. Finally, figure 9 shows the results for pure shear loading.

Different scales are necessary to plot the boundary stress variation for different shapes. Particularly, when the shapes have sharp corners, $\sigma_{t}$ shoots up rapidly. For instance, for shape $2, \sigma_{t}>50$ at $\theta=0$ (figure 5). Similar peaks are also evident for other shapes in figures 5 through 9 . All the plots are arranged in the order of increasing perimeter following the same sequence as given in figure 4 and listed in table 1 .

Two useful characteristics of the boundary stress variation along the perimeter are the mean values of $\sigma_{t}$ and $\sigma_{t(r m s)}$ defined as

$$
\sigma_{t m}=\left(\int \sigma_{t} d s / \int d s\right) ; \quad \sigma_{t(r m s)}=\left(\int \sigma_{t}^{2} d s / \int d s\right)^{1 / 2} .
$$

These mean values are tabulated in tables 4 through 8 . The first quantity $\sigma_{t m}$ gives an idea of the average perturbation in the boundary stress on account of the shape evolution. 

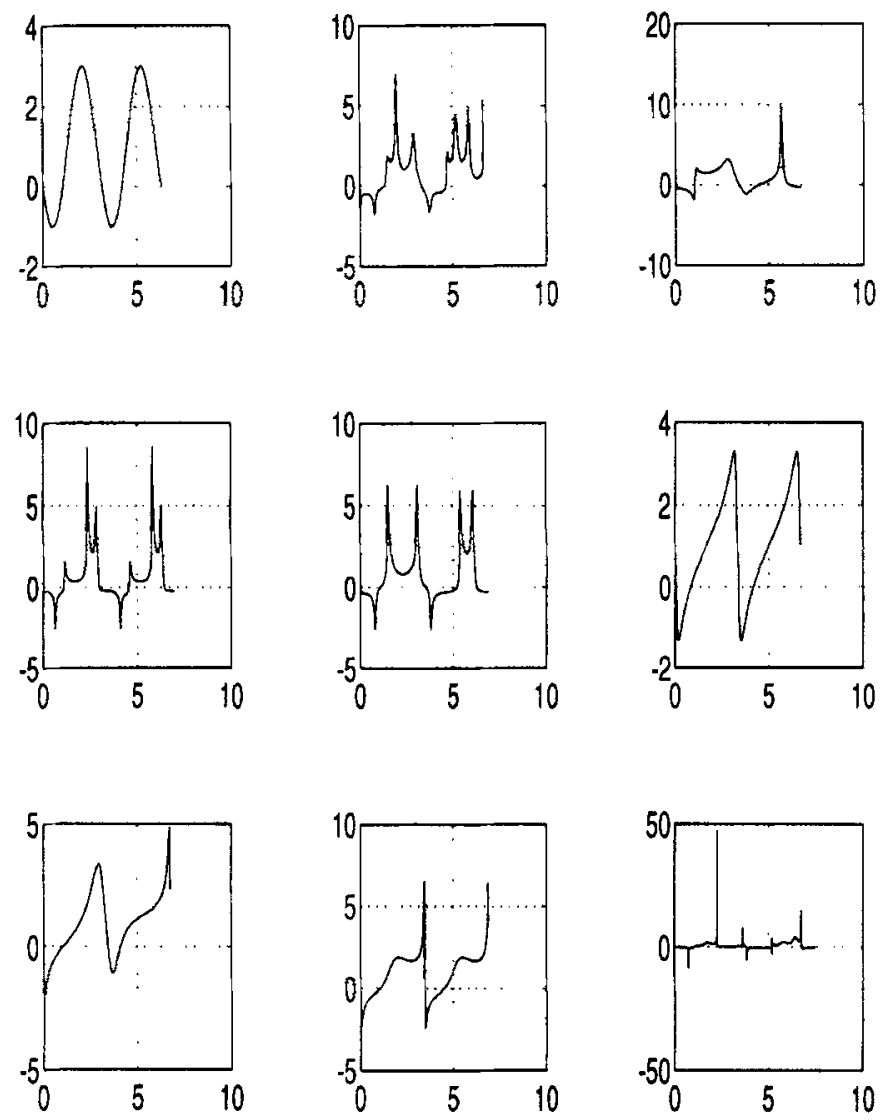

Figure 8. Stress distribution for uniaxial loading at 30 degrees.

For a circular hole in a plate $\sigma_{t m}$ equals 2,1 , and 0 for hydrostatic loading, uniaxial loading and shear loading, respectively. The second quantity $\sigma_{t(\mathrm{rms})}$ gives a qualitative measure of the perturbation in strain energy along the boundary of the irregular holes owing to shape distortion. For a circular hole, $\sigma_{t(r m s)}$ is equal to $2, \sqrt{3}, 2 \sqrt{2}$ for hydrostatic loading, uniaxial loading and shear loading respectively.

For hydrostatic loading, it is interesting to note that $\sigma_{t m}$ generally decreases with increasing perimeter, whereas $\sigma_{t(r m s)}$ does not show much variation except for shape 9 . For uniaxial loading, $\sigma_{t m}$ again exhibits a decreasing trend as shown in table 5, for $\alpha_{L}=\pi / 2$. However, when $\alpha_{L}=0$ (table 6), or $\pi / 6$ (table 7), there is no general trend for $\sigma_{t m}$. On the other hand, $\sigma_{t(r m s)}$ appears to remain close to $\sqrt{3}$, which represents the case of a circular hole (shape 1).

Finally for pure shear loading, $\sigma_{t}$ is nearly 0 whereas $\sigma_{t(\mathrm{rms})}$ increases slightly from $2 \sqrt{2}$ corresponding to the circle.

It should be borne in mind that evaluating $\sigma_{t}$ alone cannot be used to predict the mechanics of shape evolutions. A complete analysis of the stress field $\sigma_{x}, \sigma_{y}$, and $\tau_{x y}$ is necessary to discuss the global changes in strain energy. This requires the determination of the second complex potential $F$. Consequently, this paper provides only a partial analysis of the more general problem. 

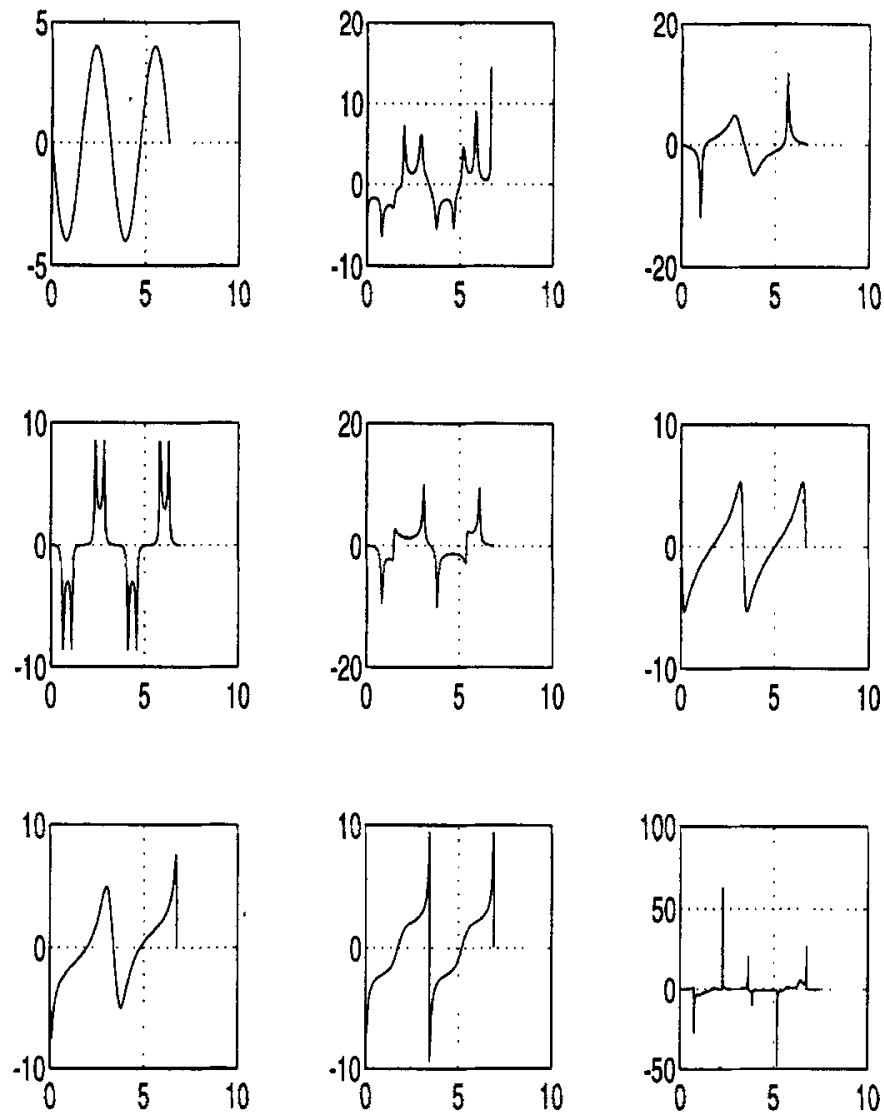

Figure 9. Stress distribution for pure shear loading.

\section{Conclusions}

Tangential stress variation along the boundary of isolated irregular holes in large plates under uniform loading was obtained using complex variables in conjunction with conformal mapping. Nine different shapes were considered including a circle and an ellipse for reference. All the 9 shapes considered had the same area but different perimeters ranging from $2 \pi$ for the circle to about 8.2 for shape 9 in figure 4 . The main theme in this paper was to understand the perturbations in the mean boundary stress $\sigma_{t m}$ and its root mean square value $\sigma_{t(r m s)}$. Significant perturbations were noted for $\sigma_{t m}$, but not for $\sigma_{t(r m s)}$. Qualitatively, it can be concluded from the results in this paper that irregular holes may change their shape if not their size by exchanging surface energy with strain energy. However, for more complete and quantitative analysis of this problem, a global stress analysis is necessary. A linear elastic analysis followed in this paper may not support the physics of change in shapes, but extending the ideas to a linear viscoelastic material is possible. Further work is necessary to understand the growth and evolution of cavities in stretched sheets to understand degradation phenomena such as stress corrosion. 
Table 4. $\sigma_{t m}$ and $\sigma_{t(r m s)}$ for hydrostatic loading.

\begin{tabular}{ll}
\hline$\sigma_{t m}$ & $\sigma_{t(r m s)}$ \\
\hline 2.0000 & 2.0000 \\
1.5412 & 1.9803 \\
1.5051 & 2.0003 \\
1.2839 & 2.0509 \\
1.3189 & 2.0412 \\
1.5136 & 2.0000 \\
1.4282 & 1.9970 \\
1.2047 & 1.9597 \\
0.7396 & 2.1911 \\
\hline
\end{tabular}

Table 5. $\sigma_{t m}$ and $\sigma_{t(r m s)}$ for uniaxial loading at 90 degrees.

\begin{tabular}{rl}
\hline$\sigma_{t m}$ & $\sigma_{t(r m s)}$ \\
\hline 1.0000 & 1.7321 \\
0.6354 & 1.4520 \\
0.7703 & 1.7272 \\
0.6556 & 1.6871 \\
0.6749 & 1.7397 \\
0.2705 & 1.9797 \\
0.2188 & 1.9528 \\
-0.0488 & 2.0136 \\
0.1806 & 1.8390 \\
\hline
\end{tabular}

Table 6. $\sigma_{t m}$ and $\sigma_{t(r m s)}$ for uniaxial loading at 0 degrees.

\begin{tabular}{ll}
\hline$\sigma_{t m}$ & $\sigma_{t(r m s)}$ \\
\hline 1.0000 & 1.7321 \\
0.8886 & 1.7215 \\
0.7402 & 1.7168 \\
0.6556 & 1.6871 \\
0.6671 & 1.7367 \\
1.2432 & 1.4046 \\
1.2042 & 1.4051 \\
1.2223 & 1.4156 \\
0.6156 & 1.7952 \\
\hline
\end{tabular}

Table 7. $\sigma_{t m}$ and $\sigma_{t(r m s)}$ for uniaxial loading at 30 degrees.

\begin{tabular}{ll}
\hline$\sigma_{t m}$ & $\sigma_{t(r m s)}$ \\
\hline 1.0000 & 1.7321 \\
0.8292 & 1.7225 \\
0.7495 & 1.7539 \\
0.6556 & 1.7673 \\
0.6695 & 1.7367 \\
1.0079 & 1.6074 \\
0.9677 & 1.6183 \\
0.9174 & 1.6298 \\
0.5123 & 1.8075 \\
\hline
\end{tabular}

Table 8. $\sigma_{t m}$ and $\sigma_{t(r m s)}$ for pure shear loading.

\begin{tabular}{rl}
\multicolumn{1}{l}{$\sigma_{t m}$} & $\sigma_{t(r m s)}$ \\
\hline 0.0000 & 2.8284 \\
-0.1251 & 2.8490 \\
-0.0011 & 2.8894 \\
0.0000 & 2.9648 \\
0.0000 & 2.8312 \\
0.0182 & 2.8918 \\
0.0195 & 2.9234 \\
0.0297 & 2.9620 \\
-0.1596 & 3.0211 \\
\hline
\end{tabular}

\section{List of symbols}

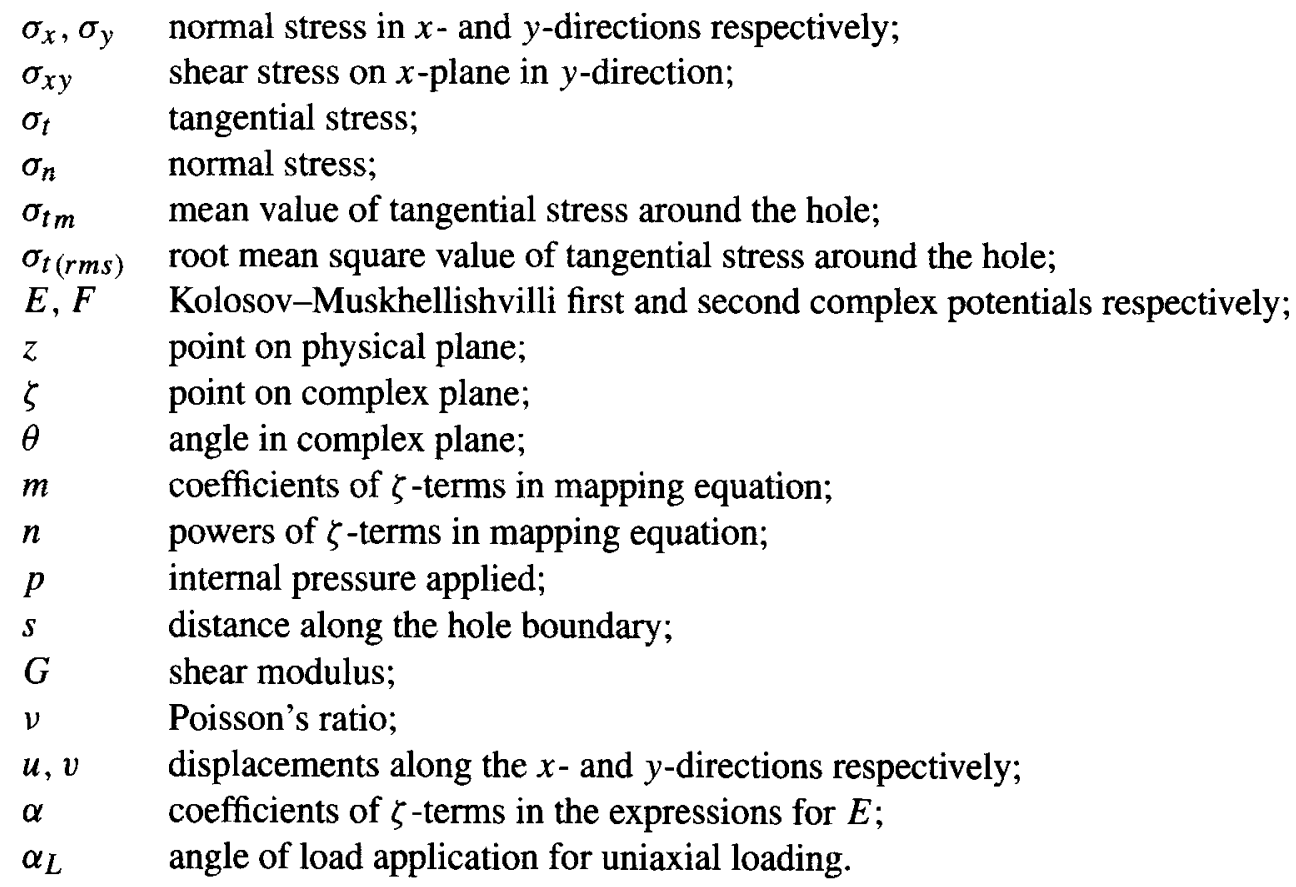




\section{References}

Carrier G F, Krook M, Pearson C E 1966 Functions of a complex variable (New York: McGrawHill)

Churchill R V 1960 Introduction to complex variables (New York: McGraw-Hill)

England A H 1971 Complex variable methods in elasticity (London: Wiley-Interscience)

Givoli D, Elishakoff I 1992 Stress concentration at a nearly circular hole with uncertain irregularities. Trans. ASME: J. Appl. Mech. 59: S65-S71

Kober H 1952 Dictionary of conformal representations (New York: Dover)

Savin G S 1962 Stress concentration around holes (New York: Pergamon)

Timoshenko S, Goodier J N 1970 Theory of elasticity (New York: Mc-Graw Hill)

Vandembroucq D, Roux S 1997a Conformal mapping on rough boundaries. I. Application to harmonic problems. Phys. Rev. E-55: 6171-6185

Vandembroucq D, Roux S 1997b Conformal mapping on rough boundaries. II. Application to biharmonic problems. Phys. Rev. E-55: 6186-6196 